\title{
Variation of perimplant biofilm induced by non surgical periodontal therapy and the use of probiotics
}

Marcello Gatti, Giovanna Costa, Tatiana Giulia Rizzati, Francesca Scandurra, Maria Sofia Rini, Pasqua Schiavone Dipartimento di Scienze Odontostomatologiche, Sezione di Microbiologia Alma Mater Studiorum, Università degli Studi di Bologna

Key words: Perimplantitis, Implant dentistry, Cultural, rRNA ibridization test, Probiotics

Variazione del biofilm perimplantare indotto da terapia parodontale non chirurgica e uso di probiotici

\section{SUMMARY}

Thanks to improved surgical tecniques the use of dental implants has increased greatly.

However, high rates of osseointegrated correctly implants, over the years are undermined by disease of bacterial etiology in the perimplant zone, especially by Gram negative anaerobes such as in gingivitis and periodontitis, in particular: Fusobacterium spp.(F.), Treponema denticola (T.d.), Tannerella forsythensis (T.f.), Aggregatibacter actinomycetemcomitans (A.a.), Prevotella intermedia (P.i.) e Porphyromonas gingivalis (P.g.). The mechanic treatment (MS) results in a reduction of the total bacterial count (TBC) and a slight change in the subgingival bacterial microflora towards the less pathogenic species and more like those of a healthy periodontium. Also the use of a probiotic in the form of buccal tablets of Lactobacillus reuteri (L.r.), as demonstrated in this study, is thought to improve and modulate the composition of plaque, as it is able to exert an inhibitory effect on oral bacteria that support caries, gingivitis, periodontal and perimplant disease with a combination of different mechanisms.

Aims

The purpose of this study was to evaluate the effect of MS associated with the use of local probiotic on variations in the composition of the subgingival microbial population in patients with perimplantitis using a microbiological quantitative test for direct rRNA hybridization of commerce and culture.

\section{INTRODUZIONE}

Negli ultimi vent'anni è aumentato sempre di più l'utilizzo della metodica implantare e le previsioni, vista anche la crescita dell'aspettativa di vita, sono di un alto numero di pazienti che dovranno essere tenuti in terapia di supporto. Controllare adeguatamente la qualità e la quantità del biofilm colonizzante i siti significherà garantire successo, dal momento che la scarsa igiene orale è stata considerata uno dei fattori di rischio più evidenti per la malattia perimplantare. Su questo fattore di rischio come del resto sull'abitudine di fumare si può agire, mentre le possibilità di controllo sono purtroppo nulle per fattori indipendenti dai nostri comportamenti come il polimorfismo genetico, l'età, la pregressa parodontopatia, la malattia metabolica.

Grazie al miglioramento delle tecniche chirurgiche negli ultimi venti anni è aumentato moltissimo l'utilizzo della metodica implantare. I dati provenienti dal Consensus Report del 2008 indicano alte percentuali di impianti inizialmente correttamente osteointegrati (11), ma nel corso degli anni minati da malattie a eziologia batterica nella zona perimplantare, soprattutto Gram negativi anaerobi come nella gengivite e parodontite $(5,6,10)$, in particolare Fusobacterium spp., Treponema denticola, Tannerella forsythensis, Aggregatibacter actinomycetemcomitans, Prevotella intermedia e Porphyromonas gingivalis in alte cariche $(4,7,9,10$, 12).

Studi microbiologici hanno dimostrato che la strumentazione meccanica (SM) determina una riduzione della conta batterica totale (CBT) e un viraggio della microflora sottogengivale verso le specie meno patogene e più simili a quelle di un parodonto sano (3).

La rimozione regolare della placca sopragengivale non ha solo l'effetto di migliorare i parametri associati all'infiammazione gengivale, ma anche quello di modulare la composizione della placca sottogengivale. Oggi si ritiene che l'introduzione di un probiotico sottoforma di compresse orosolubili a base di Lactobacillus reuteri possa migliorare e modulare la composizione della placca, in quanto la sua azione viene attribuita alla capacità di esercitare un effetto inibitorio sui batteri orali che sostengono carie, gengivite e malattia parodontale $(2,1,5,8,12)$ con una combinazione di diversi mec- canismi, inclusa la produzione di acido lattico, perossido di idrogeno, sostanze antimicrobiche e batteriocine. L'attività antimicrobica ad ampio spettro di L reuteri è considerata un'importante caratteristica che conferisce potenziale terapeutico per la prevenzione o il trattamento delle infezioni anche a livello del cavo orale in quanto converte il glicerolo in un potente composto antimicrobico ad ampio spettro, la reuterina (3-idrossi-propionaldeide), in grado di inibire la crescita di batteri Gram positivi e Gram negativi.

È un batterio ampiamente usato per le sue proprietà probiotiche, come trattamento per il riequilibrio della flora batterica gastrointestinale.

L'analisi microbiologica si avvale dell'uso dell'esame colturale tradizionale che, consentendo l'identificazione delle specie batteriche implicate, rimane il gold standard per evidenziare la presenza dei microrganismi nell'infezione perimplantare, anche se oggi è possibile utilizzare metodiche nuove come le tecniche di biologia molecolare di maggiore utilità soprattutto per ricercare alcune specie batteriche di difficile coltivazione.

Lo scopo dello studio è stato quello di valutare l'effetto di SM associato all'uso di probiotico locale sulle variazioni della popolazione batterica sottogengivale in pazienti con perimplantite utilizzando sia un test commerciale basato sull'ibridizzazione diretta dell'rRNA per la quantificazione dei batteri vitali ed esame colturale.

\section{MATERIALI E METODI}

Sono stati studiati 24 pazienti (14 donne e 10 uomini) di età compresa, le donne fra 43 e 76 anni, con una media di 63.8 +/- 10.9 anni, gli uomini tra 45 e 88 anni, con una media di 64.3 anni $+/-12.5$ anni. Tutti i pazienti avevano perso elementi dentari per parodontopatia (parodontiti ripetute fino alla perdita dell'elemento) e sostituiti con implantoprotesi. La media temporale degli impianti e della loro attività era di 11.5 anni nelle donne e di 10.7 anni negli uomini. Con l'utilizzo della sonda parodontale PCP 15 della Hu-Friedy sono stati valutati parametri clinici come BOP (sanguinamento al sondaggio), la presenza di essudato, la profondità delle tasche parodontali (PPD), il livello di attacco clinico (CAL), la mobilità (MOB). Sono stati valutati dati anamnestici come

\section{Corresponding author: Marcello Gatti}

Università degli Studi di Bologna, Dipartimento di Scienze Odontostomatologiche, Sezione di Microbiologia

Via San Vitale, 59 - 40125 Bologna - Tel.: 39 05I 2088I56 - Fax: 051 225208;

E-mail: marcello.gatti@unibo.it; marcello.gatti.bo@alice.it 
la familiarità per la malattia parodontale, la condizione di fumatori, la presenza di malattie metaboliche, di ipertensione o di altri problemi sistemici. È stata considerata la frequenza abituale della terapia di supporto (per tutti $>$ di un anno) e l'utilizzo domiciliare dei presidi per l'igiene degli spazi interprossimali.

I criteri per la non inclusione nello studio sono stati: avere assunto antibiotici nei tre mesi precedenti il prelievo, avere utilizzato collutori antibatterici nell'ultima settimana prima del prelievo, essersi sottoposti a terapia di supporto (causale) durante i tre mesi precedenti il prelievo e presenza di gravidanza.

Il campionamento microbiologico della placca sottogengivale è stato effettuato prima della SM, dopo 5, e 12 mesi.

I pazienti, dopo il primo campionamento, sono stati divisi casualmente in 2 gruppi: al gruppo A è stata effettuata solo la SM (controllo), al gruppo B (test) è stato inoltre fatto assumere giornalmente un probiotico mediante una compressa orosolubile a base di L. reuteri (GUM).

\section{Modalità di prelievo}

Le modalità utilizzate per il prelievo del materiale patologico sono state: isolare il campo operatorio, asciugare il sito individuato per il prelievo, inserire 2 coni di carta sterili (uno per l'esame colturale e l'altro per la tecnica di ibridizzazione dell'rRNA), nei siti con sondaggio $>4 \mathrm{~mm}$ per 60 ', inserire $\mathrm{i}$ coni nelle provette eppendorf sterili con terreno di trasporto per l'esame colturale e con soluzione tampone per il test molecolare.

\section{Test molecolare}

È stato utilizzato un test commerciale (PerioCheck GumSunstar) basato sull'ibridizzazione diretta dell'RNA per la quantificazione dei batteri vitali appartenenti alle specie patogene parodontali e perimplantari: A. actinomycetemcomitans (A.a.), P. gingivalis (P.g.), T. forsythensis (T.f.), e $T$. denticola (T.d.). Il limite di rivelazione per ciascuna specie patogena è di 200 cellule batteriche. I campioni per l'analisi molecolare sono stati inviati, all'istituto IAI (Instut fur Angewandte Immunologie), presso Zuchwil, in Svizzera.

\section{Esame colturale}

Per l'esame colturale i campioni sono stati osservati al microscopio a fresco per evidenziare batteri mobili, cocchi, bacilli filamentosi, ecc... e in campo oscuro per evidenziare le spirochete con ingrandimento 400x. Successivamente i campioni sono stati seminati in piastre di: Herellea agar (Biolife), Mannitol salt agar (Biolife), Blood agar horse (Biolife), Brucella agar (Biolife), CNA agar (Biolife), Schaedler selective blood agar (Biolife), Trypticase soy agar addizionato con siero di cavallo, bacitracina e vancomicina (Biolife), Gc-Lect agar (BD), MTS agar (Biolife), Chromoagar (Alfa Wasserman) e/o altri terreni selettivi preparati in laboratorio. I terreni, per la crescita di batteri aerobi, venivano poi incubati a $37^{\circ} \mathrm{C}$ per 48 ore, mentre per i batteri anaerobi i terreni venivano incubati a $37^{\circ} \mathrm{C}$ per 5 giorni in anaerobiosi utilizzando il sistema GENbag anaer (bioMérieux). Per la semina è stata utilizzata un ansa calibrata monouso sterile da $0.10 \mu l$. L'ansa veniva strisciata sulle piastre in 4 settori diversi, per ottenere una conta semiquantitativa finale di $\geq 10^{5} \mathrm{cfu}$. Le colonie sono state isolate utilizzando gli stessi terreni di crescita ed identificate con i sistemi Api (bioMérieux): Rapid ID 32, per bacilli anaerobi obbligati sia Gram negativi che Gram positivi, Rapid ANA II, per cocchi anaerobi obbligati Gram positivi, API Staph. per stafilococchi e micrococchi, Rapid ID32 Strep per streptococchi, RapID NH per Eikenella, Haemophilus, Neisseria e Aggregatibacter e API C Aux per i lieviti.

\section{RISULTATI}

Prima del trattamento tutti i pazienti studiati sono risultati positivi all'indagine microbiologica con cariche batteriche
$>10^{5} \mathrm{cfu}$. In particolare, l'esame colturale, con $\mathrm{cfu} / \mathrm{ml}>10^{5}$, è risultato positivo nel primo prelievo per: A. actinomycetemcomitans nel $41 \%$, T. forshytensis nel $69 \%$, $P$. gingivalis nel $79 \%$, Treponema denticola (T.d.) non è stata cercata con l'esame colturale, ma nel $63 \%$ dei preparati microscopici in campo oscuro sono risultati positivi per spirochete.

L'indagine molecolare ha permesso di identificare batteri con una carica che oscillava tra $10^{6}$ e $10^{7} \mathrm{cfu} / \mathrm{ml}$, evidenziando, nel primo prelievo, una positività per: A. actinomycetemcomitans nel $21 \%$, T. forshytensis nel $83 \%$, P. gingivalis nel $71 \%$ e T. denticola nel $67 \%$.

Suddividendo i pazienti casualmente in 2 gruppi dopo SM: gruppo A (controllo) e gruppo B (test) e ripetendo i prelievi a distanza di 5 e 12 mesi si è visto, con l'esame colturale, che: il gruppo A è risultato positivo per $A$. actinomycetemcomitans nel $17 \%$ nel secondo prelievo e nel $28 \%$ nel terzo prelievo, $T$. forshytensis nel $34 \%$ nel secondo prelievo e $45 \%$ nel terzo prelievo, $P$. gingivalis nel $28 \%$ nel secondo prelievo e nel $60 \%$ nel terzo prelievo, le spirochete sono state osservate al microscopio in campo oscuro, nel $33 \%$ nel secondo prelievo e $65 \%$ nel terzo prelievo.

Il gruppo B è risultato positivo per A. actinomycetemcomitans nel $2 \%$ nel secondo prelievo e nel $12 \%$ nel terzo prelievo, T. forshytensis nel $14 \%$ nel secondo prelievo e $25 \%$ nel terzo prelievo, $P$. gingivalis nel $14 \%$ nel secondo prelievo e nel $40 \%$ nel terzo prelievo, le spirochete sono state osservate al microscopio in campo oscuro, nel 3\% nel secondo prelievo e $48 \%$ nel terzo prelievo.

L'indagine molecolare condotta sui 2 gruppi di studio A (controllo) e B (test) ha evidenziato nel gruppo A: A. actinomycetemcomitan nel $22 \%$ nel secondo prelievo e $32 \%$ nel terzo prelievo, $T$. forshytensis nel $44 \%$ nel secondo prelievo e nel $70 \%$ nel terzo prelievo, $P$. gingivalis nel $48 \%$ nel secondo prelievo e nel $65 \%$ nel terzo prelievo, $T$. denticola nel $45 \%$ nel secondo prelievo e nel $70 \%$ nel terzo prelievo; nel gruppo B: sono state evidenziate percentuali più basse: $A$. actinomycetemcomitan nel 12\% nel secondo campione e nel $25 \%$ nel terzo, T. forshytensis nel 30\% nel secondo prelievo e nel $55 \%$ nel terzo prelievo, $P$. gingivalis, nel $38 \%$ nel secondo prelievo e $58 \%$ nel terzo prelievo, $T$. denticola nel $33 \%$ nel secondo prelievo e nel $65 \%$ nel terzo prelievo.

Tali risultati sono riportati nella Figura I.

Le conte batteriche totali (CBT) del test molecolare rilevate a cinque mesi da pre-SM hanno mostrato un aumento del $19 \%$ nel gruppo A, mentre sono diminuite del $2 \%$ per il gruppo B; a 12 mesi da pre-SM la CBT per il gruppo A è aumentata del 14\%, mentre per il gruppo B è diminuita del 14\%.

Le CBT, espresse in percentuali, dei 4 batteri parodontopatogeni trovati con il test molecolare, a cinque mesi da pre-SM sono diminuite del $64 \%$, a 12 mesi del $35 \%$ per il gruppo B. Nel gruppo A, invece, a 5 mesi da pre-SM si nota una riduzione del $13 \%$ e a 12 mesi del 10\%, come riportato nella Figura II.

L'esame colturale per A.a, T.d., P.g. e T.f. ha avuto lo stesso andamento di quello molecolare, ma con percentuali più basse. Prendendo in considerazioni solo i pazienti del gruppo $\mathrm{B}$, l'esame colturale ha messo in evidenza altre specie microbiche aerobie nel primo, secondo e terzo prelievo: P. aeruginosa (rispettivamente $12 \%, 9 \%$ e $7 \%$ ), K. pneumoniae (rispettivamente $15 \%, 10 \%$ e $6 \%$ ), E. coli (rispettivamente $20 \%, 15 \%$ e $5 \%$ ), Enterobacter spp. (rispettivamente 10\%, $8 \%$ e $4 \%$ ), S. epidermidis (rispettivamente $7 \%, 5 \%$ e $5 \%$ ), $S$. aureus (rispettivamente $10 \%, 9 \%$ e $8 \%$ ), Streptococcus spp. (rispettivamente $25 \%, 22 \%$ e $20 \%$ ), C. albicans (rispettivamente $5 \%, 3 \%$ e $1 \%$ ), come riportato nella Figura III.

L'esame colturale per batteri anaerobi ha evidenziato anche la presenza, nei tre campioni dei pazienti del gruppo B di: $L$. reuteri (rispettivamente $0 \%, 50 \%$ e $30 \%$ ), Capnocytophaga spp. (rispettivamente $10 \%, 4 \%$ e $8 \%$ ), Campylobacter spp. (rispettivamente $12 \%, 6 \%$ e $9 \%$ ), P. intermedia (rispettivamente $25 \%, 10 \%$ e $16 \%$ ), Actinomyces spp. (rispettivamente $22 \%, 8 \%$ e $12 \%$ ), Fusobacterium spp. (rispettivamente $20 \%$, $12 \%$ e $15 \%$ ), come riportato nella Figura IV. 


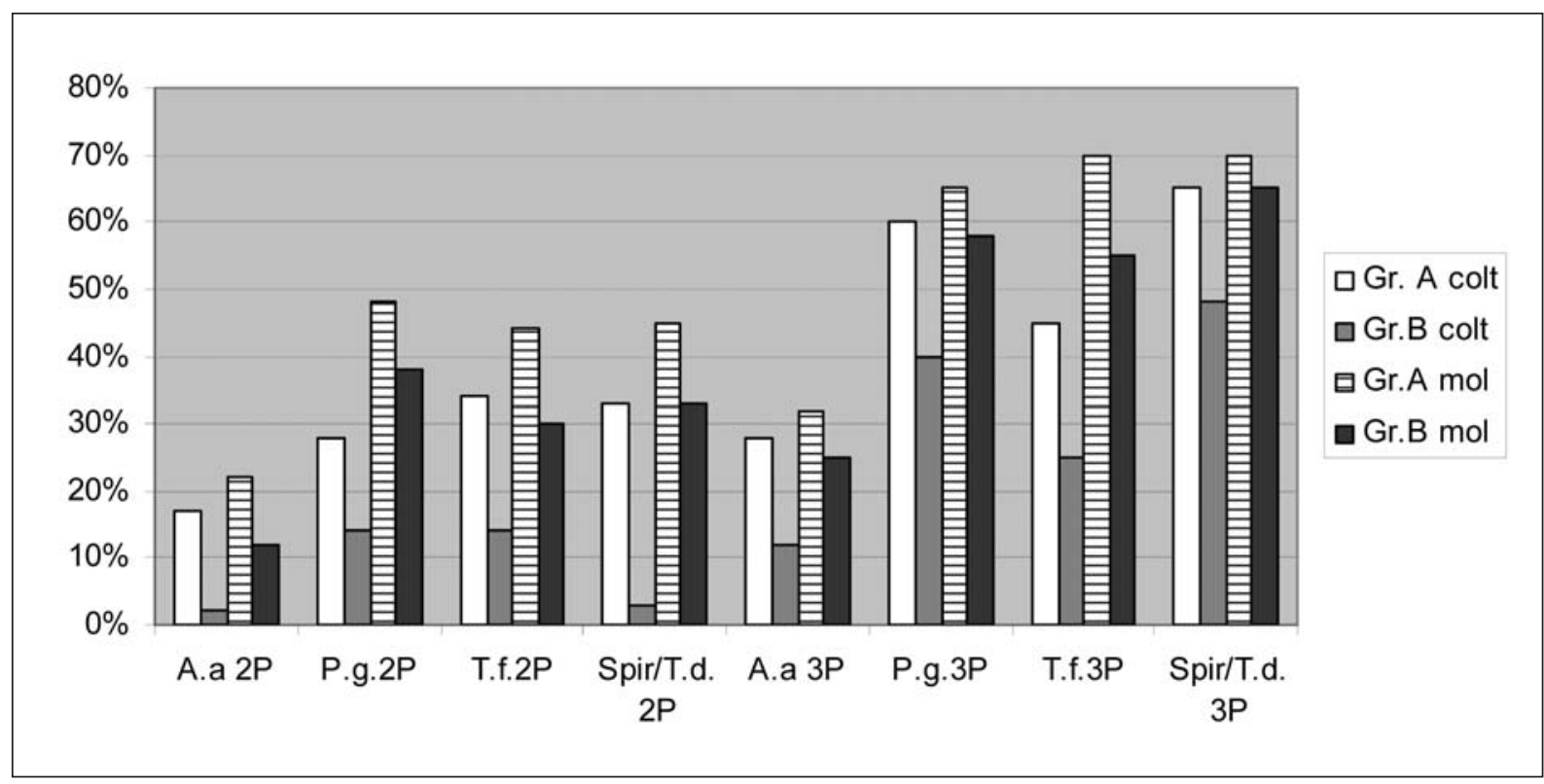

Figura I. Percentuali di presenza dei 4 batteri parodontopatogeni trovati nel secondo (2P, dopo 5 mesi da SM) e terzo prelievo (3P dopo I 2 mesi da $\mathrm{SM})$, nei due gruppi di studio (A controllo e B test) con il test molecolare ed esame colturale.

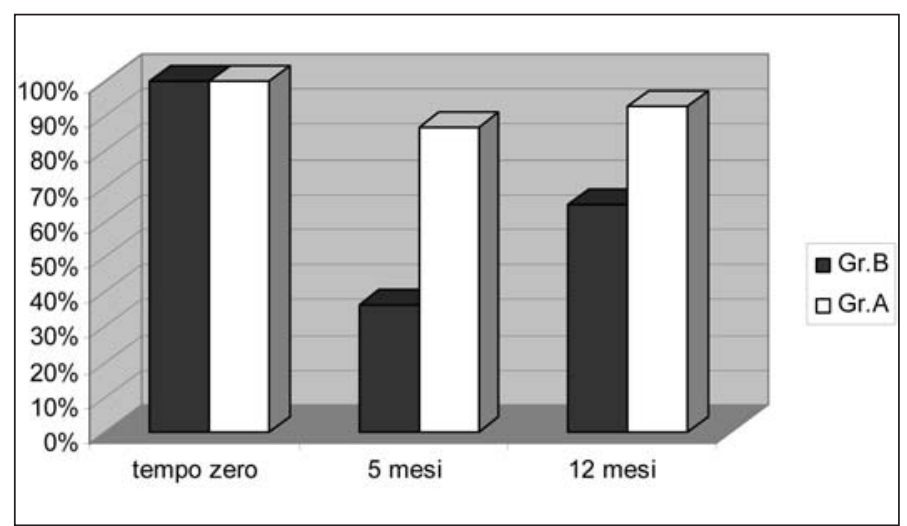

Figura II. Confronto delle percentuale delle CBT dei 4 batteri parodontopatogeni trovati con il test molecolare a tempo zero, dopo 5 e 12 mesi da pre-SM sia nel gruppo di controllo $A$ che nel gruppo test $B$.

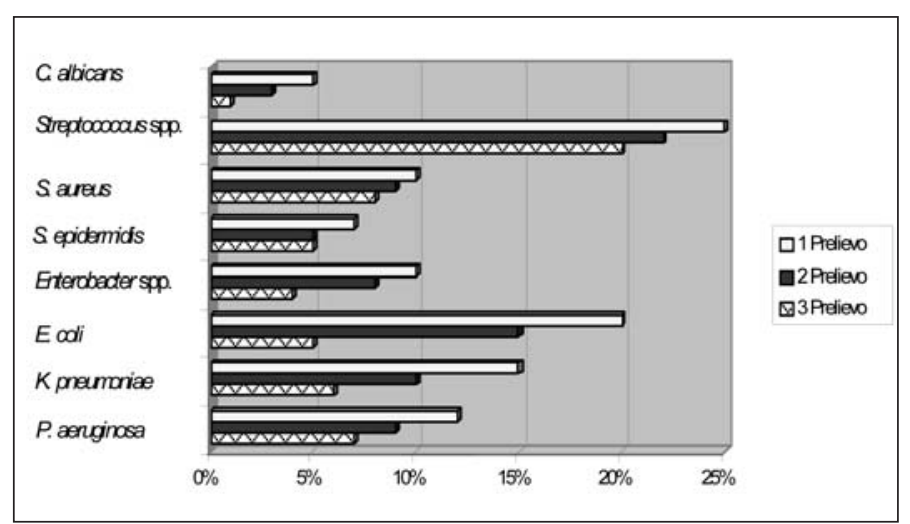

Figura III. Percentuali di specie microbiche aerobie facoltative trovate nei tre prelievi dei pazienti di gruppo $B$.

\section{DISCUSSIONE}

I risultati, ottenuti con il test molecolare, dimostrano che $T$. denticola, $P$. gingivalis, e $T$. forsythensis sono batteri etiopatogeneticamente correlati con tale malattia. I risultati del- l'esame colturale hanno dimostrato la presenza di 18 specie microbiche diverse, ma le percentuali trovate sono inferiori a quelle del test molecolare. In particolare le spirochete, sono state osservate al microscopio in campo oscuro e successivamente identificate come $T$. denticola con il test di ibridizzazione nel $63 \%$ dei pazienti oggetto del presente studio. I dati ottenuti indicano che il test molecolare è risultato più sensibile e specifico rispetto all'esame colturale. La diagnosi molecolare risulta particolarmente importante per $T$. denticola, microrganismo di difficile coltivazione.

Da questi dati preliminari si mette in evidenza che l'esame colturale, con tutti i suoi limiti, evidenzia la composizione polimicrobica presente nella zona perimplantare dei pazienti studiati ed alcuni microrganismi che in certe situazioni possono essere responsabili di infezioni acute e/o croniche. Il test molecolare ha il vantaggio di fornire risultati in tempi brevi nonché precise conte e percentuali. La placca dentale, se non attentamente controllata, può provocare condizioni patologiche. Avere la possibilità di agire sulla modulazione dell'associazione batterica è molto importante, specialmente nella zona perimplantare che strutturalmente è più aggredibile e meno duttile di quella parodontale. L'indagine micrbiologica, se correttamente interpretata, può intercettare dei parametri che rappresentano segnali di situazioni cliniche più o meno conclamate. Avere a disposizione i risultati microbiologici può aiutare l'intero team odontoiatrico nella motivazione del paziente per seguire un corretto stile di vita che unito ad adeguate manovre igieniche domiciliari e al rispetto delle scadenze individualizzate della terapia di supporto (dettate da conte e percentuali batteriche reperite) conduce ad un miglioramento della salute non solo orale, ma anche generale. Alla luce degli incoraggianti risultati, microbiologici e clinici, ottenuti nel gruppo $\mathrm{B}$ (assunzione di probiotico) più che in quello di controllo, sembrerebbe opportuno continuare la sperimentazione adoperandosi nella ricerca di strategie che portino, quando necessario, ad un sovvertimento dei rapporti di forza all'interno della comunità batterica.

I risultati ottenuti indicano che dopo cinque mesi la CBT risa- 


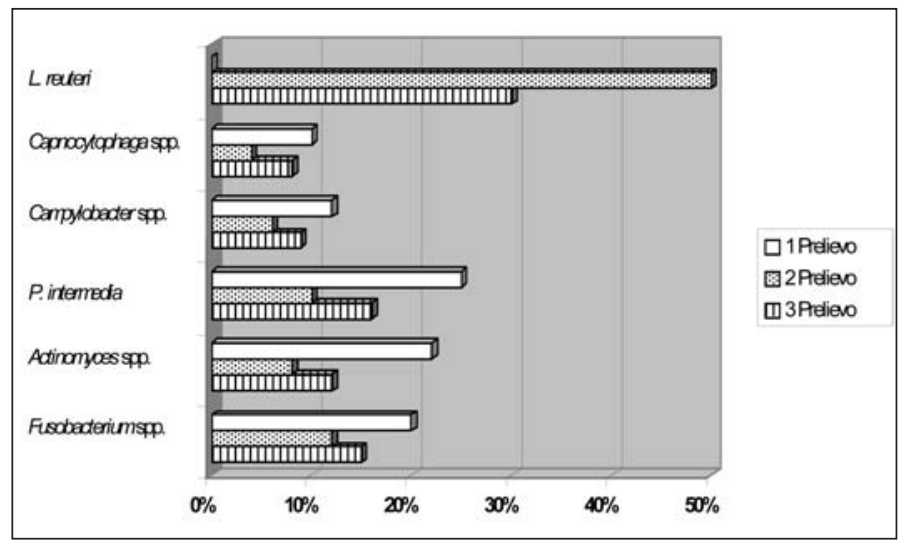

Figura IV. Percentuali di specie batteriche anaerobie trovate nei tre prelievi dei pazienti di gruppo $B$.

le del 50\%. L'uso del probiotico, utilizzato giornalmente nei pazienti del gruppo B, riduce la crescita da parte dei batteri anaerobi, favorendo il mantenimento di batteri aerobi/anaerobi non coinvolti direttamente nella malattia. L. reuteri si è dimostrato efficace se assunto in modo costante a media distanza (5 mesi) in quanto mantiene i batteri parodontopatogeni intorno al $40 \%$, diversamente dal gruppo di controllo. Prolungando nel tempo l'assunzione giornaliera del probiotico a 12 mesi si vede che il valore dei batteri parodontopatogeni, nel gruppo B aumenta fino ad arrivare al $60 \%$, mentre nel gruppo A, di controllo, raggiunge circa il $90 \%$.

In conclusione, l'igiene domiciliare continua in associazione con il probiotico, somministrato a cicli regolari di cinque mesi possono garantire il controllo della gengivite/perim- plantite. Si può ipotizzare, inoltre, che l'igiene professionale a cinque mesi in corso di terapia probiotica possa consentire il contenimento della popolazione parodontopatogena.

\section{BIBLIOGRAFIA}

1. Asikainen $\mathrm{O}$, et al. In vitro growth inhibition of periodontitisassociated species by Lactobacillus reuteri. Presented at the Anaerobes Congress. July 2006, USA.

2. Caglar E, et al. Bacteriotherapy and probiotics' role on oral healt. Oral Dis 2005; 11: 131-7.

3. Costa G, Rizzati TG, Scandurra F, Comastri C, Gatti M. Esame colturale e RT-PCR nella valutazione della modulazione del biofilm perimplantare indotta da terapia parodontale non chirurgica e probiotici. Atti del XVI Congresso Nazionale di Parodontologia SIDP. Bologna 4-6 marzo 2010.

4. Henderson B, Wilson M, Sharp L, Ward JM. A. Actinomycetemcomitans. J Med Microbiol 2002; 51 (12): 1013-20.

5. Krasse P, et al. Decreased gum bleeding and reduced gingivitis by the probiotic Lactobacillus reuteri. Swed Dent J 2006; 30: 55-60.

6. Mombelli A. Microbiology of the dental implant. Adv Dent Res 1993; 7 (2): 202-6

7. Mombelli A, Van Oosten MAC, Schürch E, Lang NP. The microbiota associated with successful or failing osseointegrated titanium implants. Oral Microbiol Immunol 1987; 2, 145-51.

8. Nikawa $\mathrm{H}$, et al. Lactobacillus reuteri in fermented bovine milk decreases the oral carriage of mutans streptococci. Int J Foof Microbiol 2004; 95: 219-23.

9. Rodemburg JP, van Winkelhoff AJ, Winkel EG, et al. Occurrence of Bacteroides gingivalis, Bacteroides intermedius and A. actinomycetemcomitans in severe periodontitis in relation to age and treatment history. $J$ Clin Periodontol 1990; 17 (6): 392-9.

10. Slots J, Ting M, Adams DA, et al. A actinomycetemcomitans and Porphyromonas gingivalis in human periodontal disease: occurrence and treatment. Periodontol 2000. 1999; 20: 82-112.

11. Smith DE, Zarb G. Criteria for success of osseointegrated endosseous implants. J Prosth Dent 1989; 62: 567-72.

12. Van Winkelhoff AJ, Wolf JW. Actinobacillus actinomycetemcomitansassociated peri-implantitis in an edentulous patient. A case report. $J$ Clin Periodonto 2000; 27 (7): 531-5 